\title{
Spanish Translation for Astronomy Outreach
}

\author{
James M. Lattis ${ }^{1}$ \\ UW Space Place, Univ. of Wisconsin-Madison Dept. of Astronomy
}

\section{Introduction}

Astronomy and space science outreach to the Hispanic community in Madison has taken many forms and presents many challenges. With support from WSGC, UW Space Place has experimented with a wide variety of Spanish-language outreach, including both bi-lingual (English presentation with simultaneous translation into Spanish) and Spanish-only interactive sessions, lectures in Spanish, star parties with Spanish interpreters present, community science "fairs" targeted for the Spanish-speaking community, and Spanish translations of exhibit materials. (See proceedings paper from last year.) In addition to the program development itself, each of these types of outreach requires Spanish-language promotional materials, support materials (e.g. agendas and event maps), and supplementary materials, such as take-home brochures. Thus, the simple-sounding process of translation itself arises constantly, although in different contexts, from beginning to end. I report here on the particular matter of translating outreach material of various sorts into Spanish. The appendix to this report contains examples of this translation work, funded entirely by WSGC.

\section{Types of Materials and Translators}

When it comes to translation, there is an important distinction between promotional/support materials and the program content and take-home materials. The first rarely depend on any scientific content (except perhaps as illustrations) and are intended only to draw attention to the program, portray it as appealing, and make the logistics (such as place and time) clear. On the other hand, program content and supplementary materials are far more demanding of the writer or translator. As an educational enterprise, it is very important that a scientific discussion should introduce appropriate terminology in Spanish for the objects, processes, etc. that are part of the lesson. It is not hard to imagine how a simple dictionary lookup of technical terms could lead to Spanish expressions that diverge from what would be normal usage by Spanish-speaking scientists.

Items like advertisements and agendas, then, can be composed or translated into Spanish by nearly any competent writer with command of Spanish. But reliable scientific language requires a translator with some significant background in science, and they can be hard to locate.

\section{Recommendations}

We have found that the talents of foreign graduate students and post-doctoral fellows in the University of Wisconsin System are a potentially excellent pool of scientifically literate native Spanish speakers who are often willing to do translation on a contract basis. A centralized registry to match up providers with those in need of such services could be a useful contribution to the science outreach community across the entire state.

1 The author wishes to acknowledge the essential support of the Wisconsin Space Grant Consortium in carrying out the work described here. 
Únase a nosotros para un día de

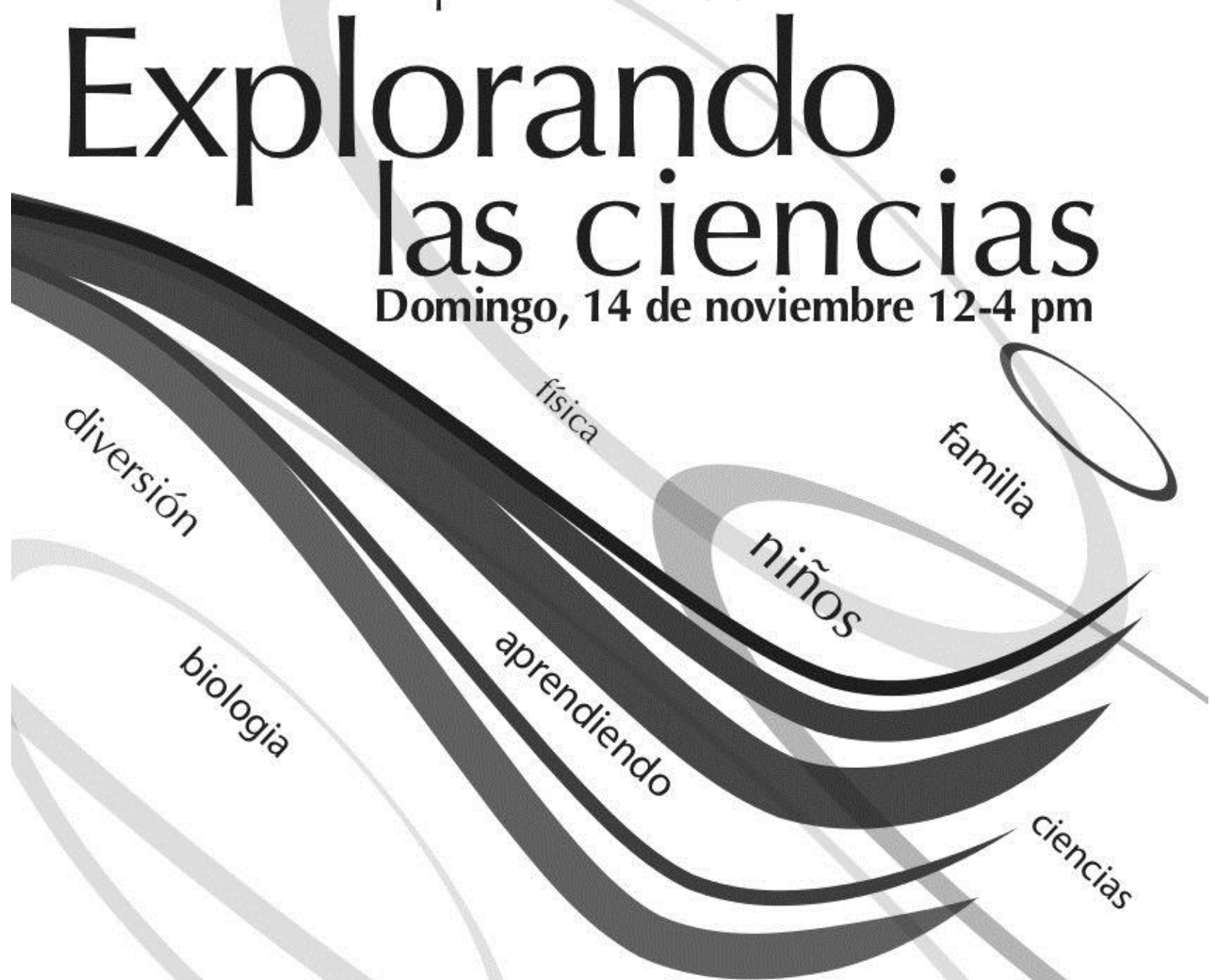

Explorando las Ciencias es un día para que toda la familia se divierta y aprenda acerca de las actividades científicas en la Universidad de Wisconsin. Habrá actividades prácticas para niños de todas las edades además de de científicos que responderán a tus preguntas.

- Aprender a usar las herramientas científicos.

- Descubre cómo hacer que un centavo parezca plata.

- Mira un relámpago en una esfera de cristal.

- Hablar con científicos que construyeron IceCube, el telescopio bajo el heilo del Polo Sur.

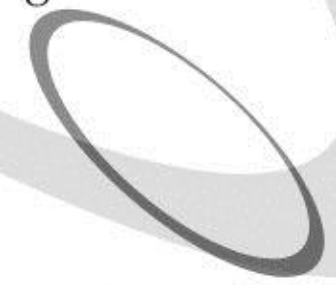

Space Place, en el Villager Mall, 2300 South Park Street 


\section{Una Parte}

de la Historia del Espacio

\section{Loseta de Protección}

\section{Térmica del Trôsbordador} Espacial de lo! NASA

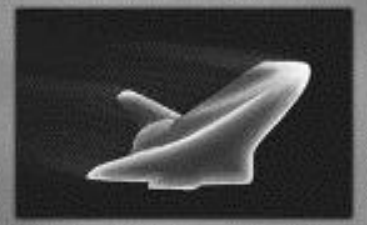

¿Cómo funcionan las losetas de protección térmica?



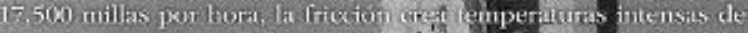
3000 grados Fahrenhicit (aproximkaliniente 1650 grados Celsius). Que protege al trasbordador pain ne quemarse' Losetas! Cada trashordador tiene mis de 21,00000 s.

¿Qué significan los números?

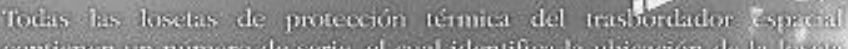
camtienen un numeno de serie, el cual identifica la miscacion de la lonelh en d orbitador. Cada loseta es unica y hecha para que encale ch una localzzación especifica en el orbitador tal como un gran rompecabezas:

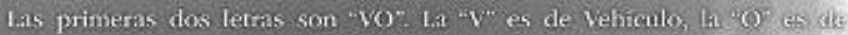
orbitador. Los seis numeros (191007) dam la ubicación en el vehiculo itin esca loscta, significa la parte inferior de has alas.
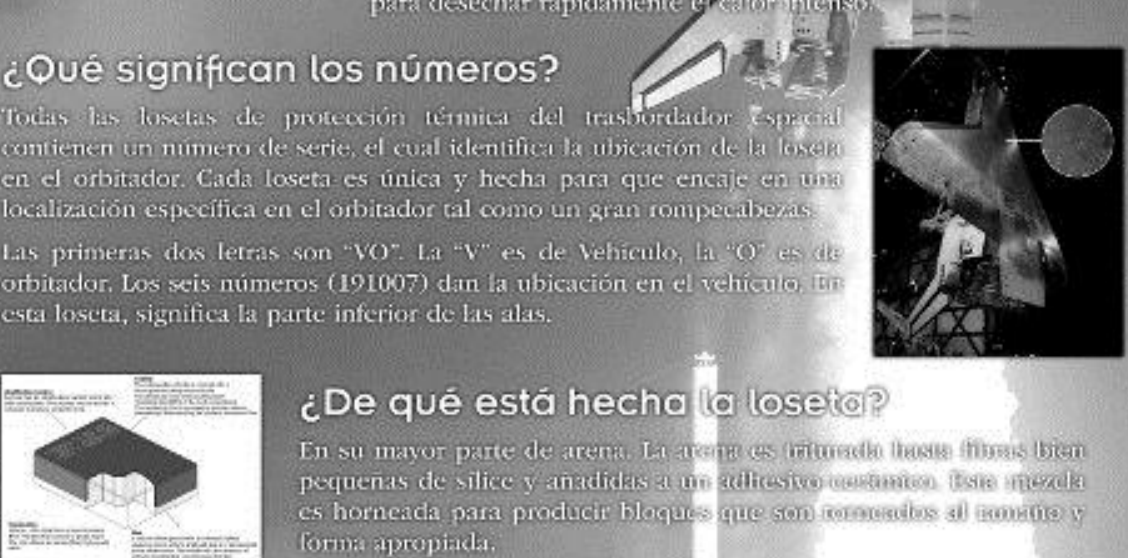

\section{¿De qué está hecha lio losetâ?}

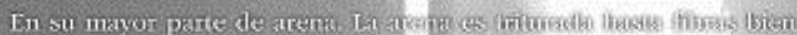

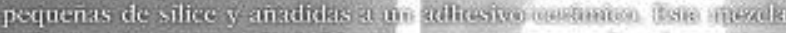

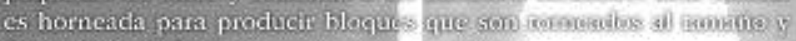

\section{¿Cuánto pesa la loseta?}

Tome cada uno de los tres cjemplas de losen de abaje ves sil puectib

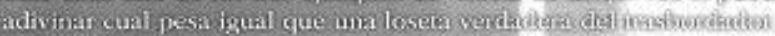




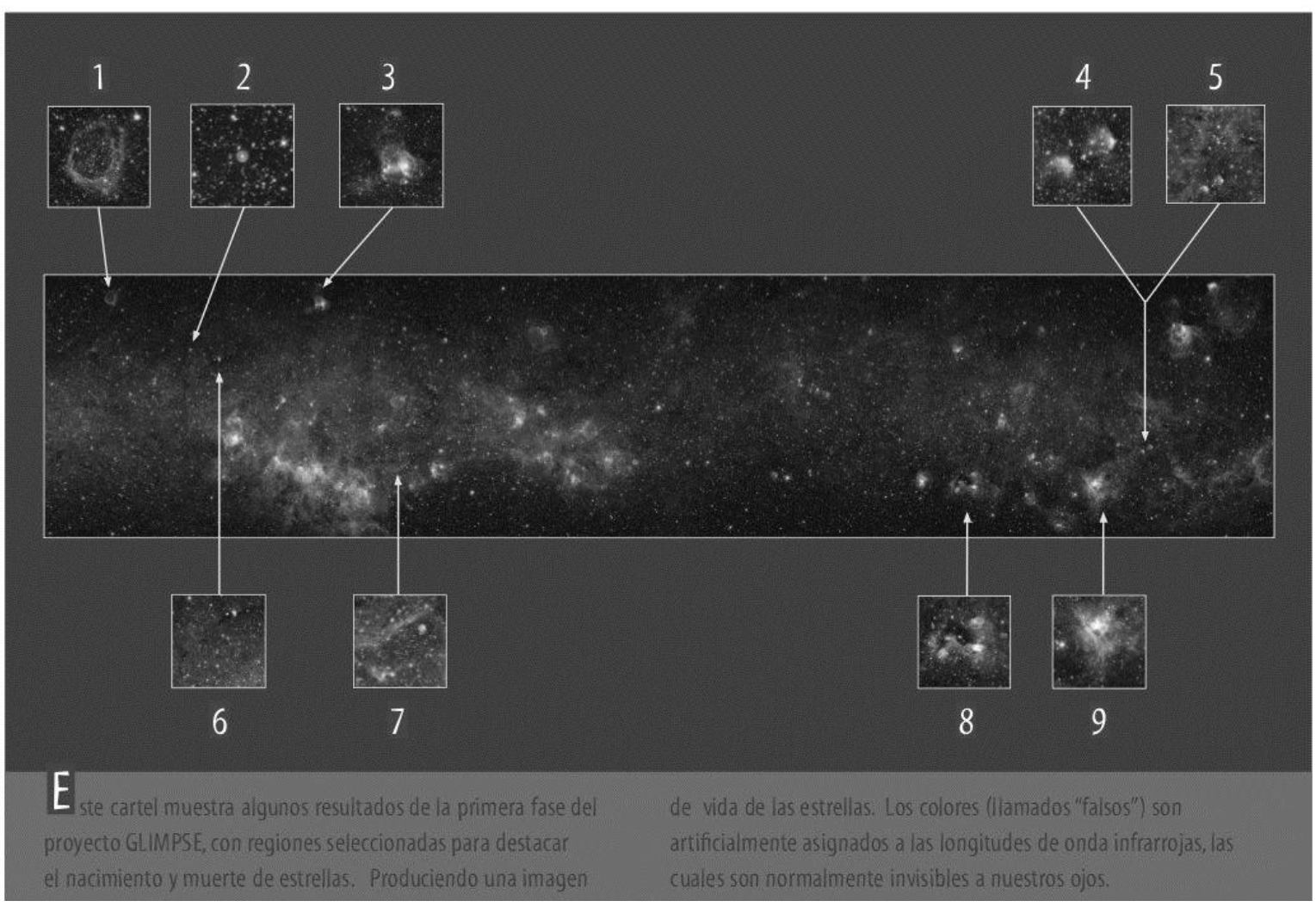

1 Región HII (pronunciado "H-dos") - una nube de 1 gases tibios y polvo del cual se forman las estrellas jóvenes. Esta imagen muestra que el gas es soplado para formar una burbuja. El soplo viene de los vientos estelares de una estrella masiva joven.

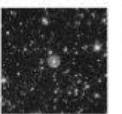

2 Nébula planetaria - una bola creciente de gas

2 expulsada por una estrella moribunda, cuya mas habia sido similar a la de nuestro sol.

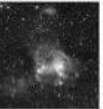

3 Una región de formación de estrellas con una región 3. asociada de HII.

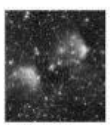

4 Una región de la Vía Láctea en la cual se están

4 formando estrellas. El color rojo falso es causado por partículas de polvo "excitadas" por la luz ul travioleta emitida de estrellas masivas jóvenes.

Nubes oscuras filamentosas, de las cuales se están formando estrellas. Regiones como ésta provocan preguntas importantes acerca de las condiciones (tales como la densidad, la temperatura y la presión) que inician el colapso de estas nubes para formar estrellas nuevas.



Nubes oscuras filamentosas de gas y polvo de las cuales estrellas jóvenes se formarán eventualmente.

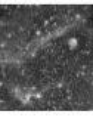

7 Los restos de una estrella que explotó como una supernova.

8 Otra del gran número de regiones donde estrellas 0 se forman. En regiones donde estrellas se forman hay "protoestrellas" objetos que están creciendo y que pronto serán estrellas. Las protoestrellas generan efusiones supersónicas, las cuales empujan las nubes de gas y polvo alrededor. El color verde falso muestra esta interacción.

g Una región muy masiva de formación de estrellas. 


\section{¿Qué eS GLIMPSE?}

os Astrónomos de la Universidad de Wisconsin

L dirigen el proyecto GLIMPSE (Estudio Extraordinario Legado Galáctico del Plano Medio por Infrarrojo), el cual usa el Telescopio Espacial Spitzer (TES) de NASA. Lanzado al espacio en 2003, el TES es la última nave espacial en el programa de Grandes Observatorios de NASA. El programa también incluye el Telescopio Espacial Hubble (TEH). EI TES es diferente del TEH, porque observa por ondas infrarrojas del espectro, mientras el TEH observa por ondas visibles.

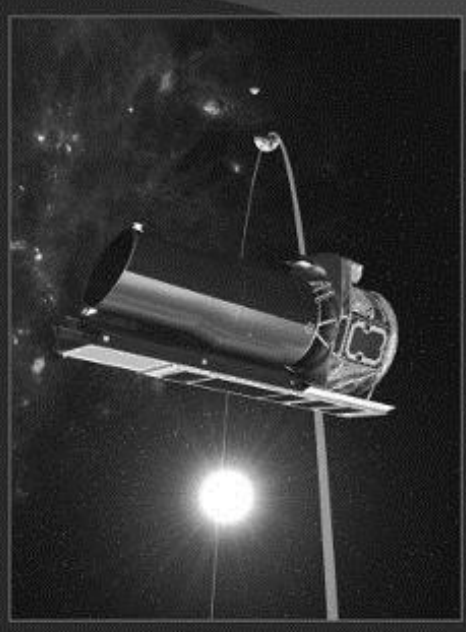

EI TES es el telescopio infrarrojo más grande

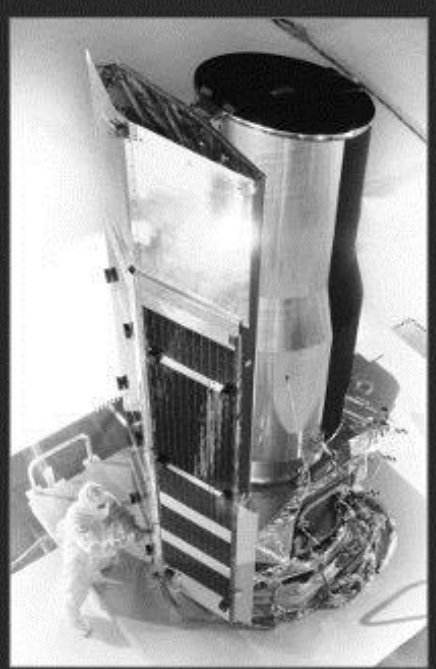

que jamás se ha lanzado al espacio. Siendo que objetos tibios emiten mucha radiación infrarroja, se tomaron precauciones para proteger el TES de interferencia de ondas no deseadas. La nave espacial es enfriada a temperaturas que acercan al cero absoluto $\left(-273^{\circ} \mathrm{C} 0-459^{\circ} \mathrm{F}\right)$, para que el telescopio no detecte su propia radiación. Lleva una cubierta grande para protegerlo del sol. EI TES fue colocado en una órbita alrededor del sol para evitar otra fuente grande de radiación no deseada, es decir, la tierra. 


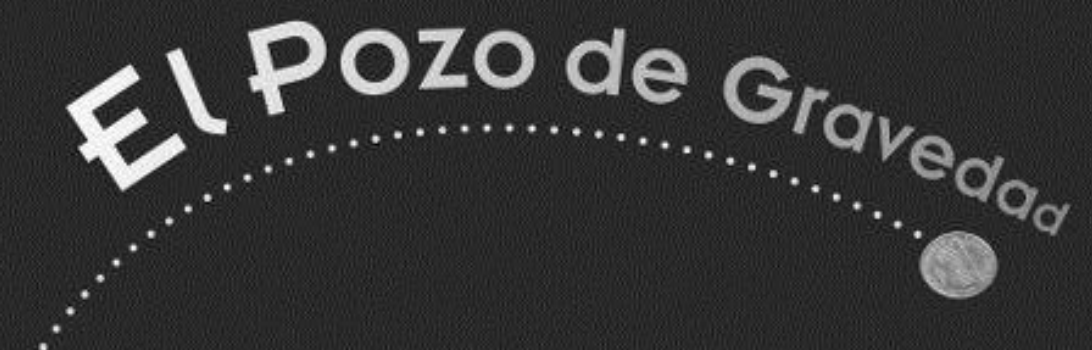

$\therefore \quad$ Pon una moneda en uno de los lanzadores.

$\therefore$ Intenta lanzar dos monedas de ambos lanzadores al mismo tiempo.

¿Siguen todas las monedas el mismo camino, y mueven a la misma velocidad? ¿Qué altera su manera de viajar?

Cuando lanzas tu moneda, tu la pones en moción. Tu moneda quisiera viajar en una línea recta con una velocidad constante. Esto se llama la INERCIA.

Tu moneda no logra continuar en una linea recta. Esto resulta porque la GRAVEDAD ejerce su efecto en tu moneda. La gravedad es la fuerza que hace que los objetos caigan al piso.

Los efectos combinados de INERCIA y GRAVEDAD hacen que la moneda viaje en un camino casi elíptico atrededor del pozo.

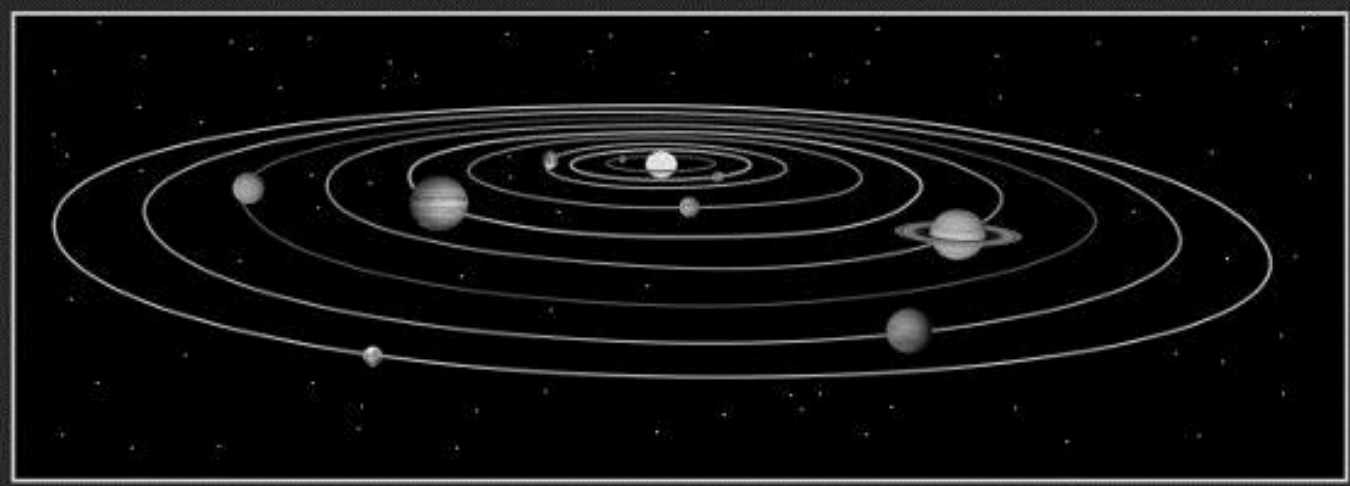

La forma que tiene el Pozo de Gravedad imita el efecto de la gravedad del sol sobre un planeta en órbita alrededor del sol.

La fricción y la resistencia del aire hacen que las monedas en el Pozo de Gravedad pierdan energia con cada vuelta, causando que caigan al fondo. En el espacio, hay poca fricción para disminuir la velocidad de los planetas. 

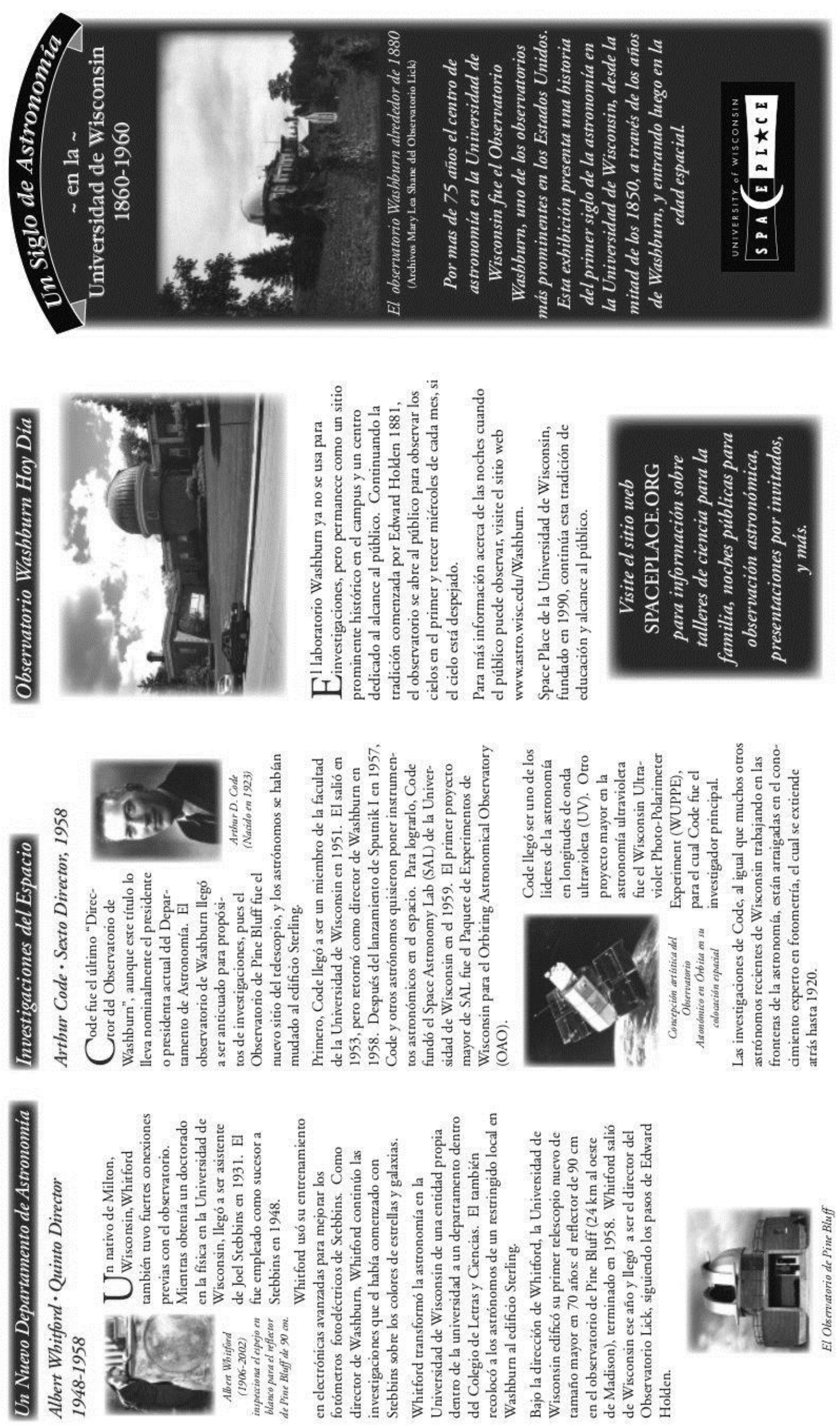

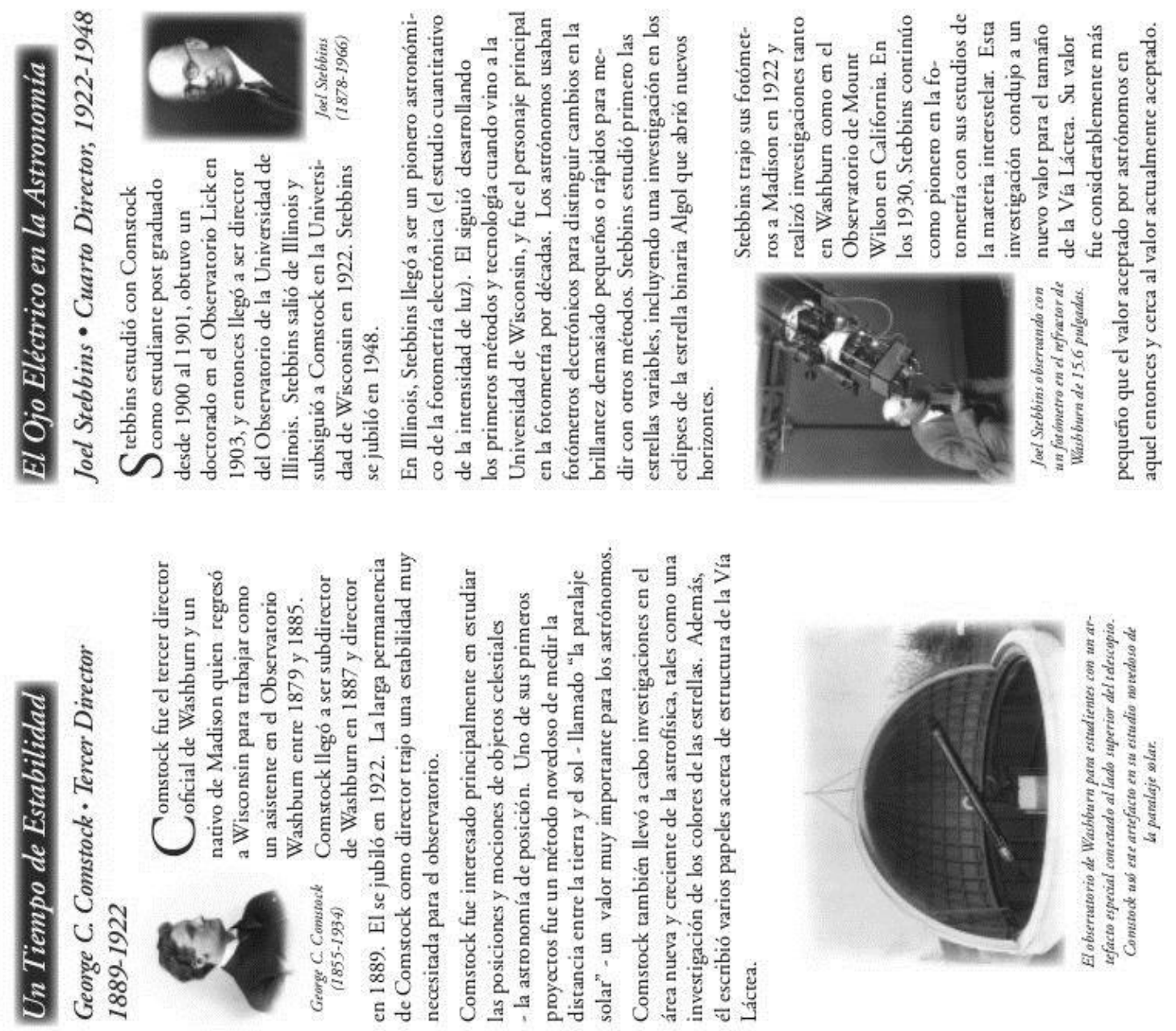

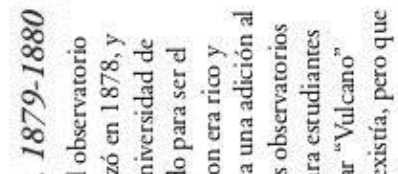
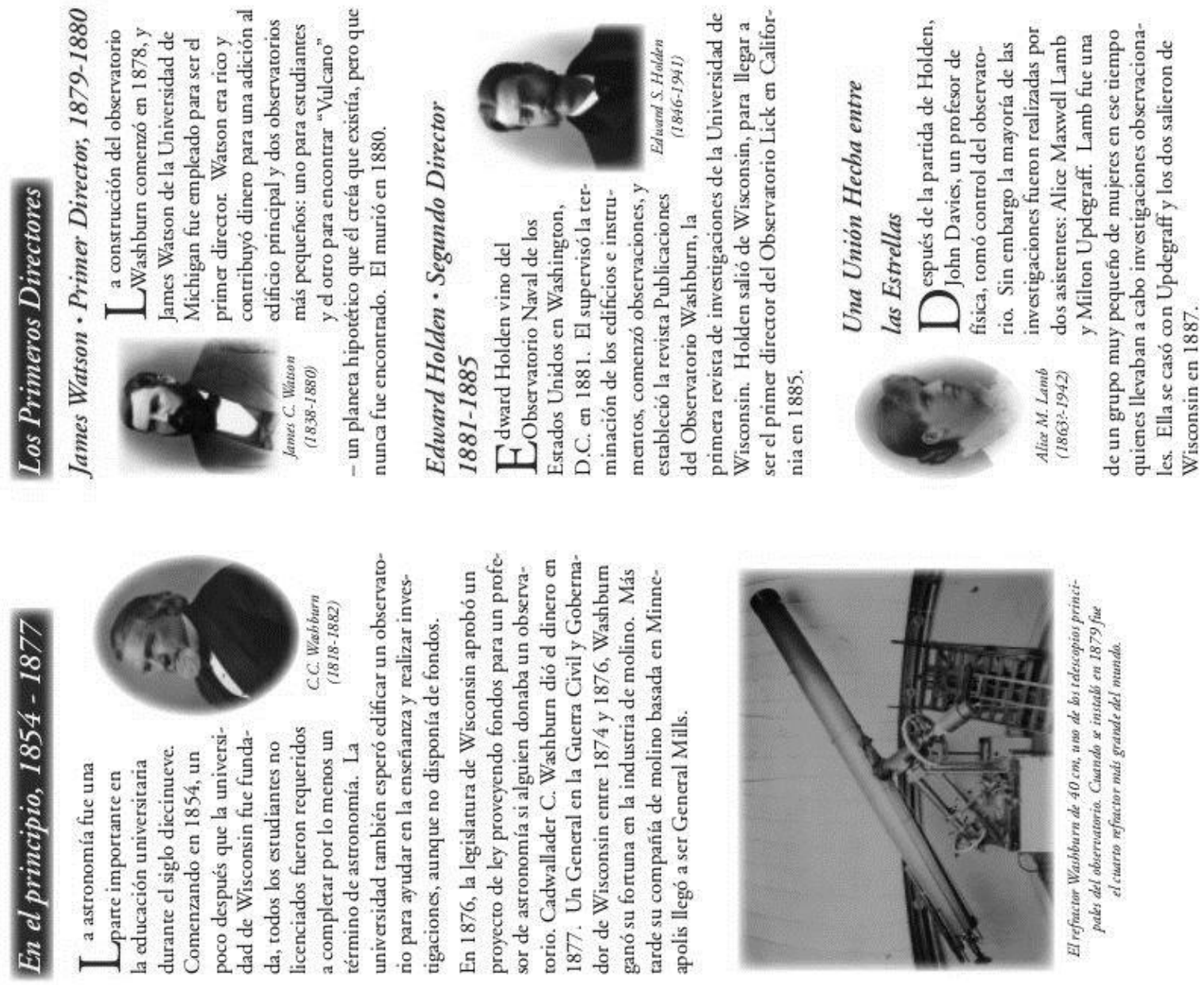
Peso y Masa:

¿Cuál es la diferencia?

Masa es una medida de cuánta materia contiene un objeto. Si viajamos a otro planeta, todavía tenemos la misma cantidad de masa pero nuestro peso cambia.

Peso es una medida de la atracción de gravedad entre usted y el planeta en el que está parado. Su peso cambiará dependiendo de la masa del planeta y a qué distancia está usted del centro de dicho planeta. 


\section{Su Peso en Otros Planetas}

Mi peso en la Tierra es lbs.

¿Qué sabe de cada uno de los planetas? ¿Qué tanta masa tiene y qué grande (qué distancia hay del centro del planeta a la superficie) es?

Prediga si su peso será mayor o menor en cada planeta. Luego haga los cálculos para cada planeta y vea si su predicción es correcta.

\begin{tabular}{|c|c|c|}
\hline & $\begin{array}{l}\text { Prediga } \\
\text { (marque uno) }\end{array}$ & Real \\
\hline MERCURIO & $\uparrow \quad \sqrt{t}$ & \\
\hline VENUS & \} $\quad\}$ & \\
\hline LUNA & 个 $\quad \sqrt{ }$ & \\
\hline MARTE & 仓 $\quad \checkmark$ & \\
\hline JUPITER & ఏ $\quad \sqrt{ }$ & \\
\hline SATURNO & $\Uparrow \quad \sqrt{ }$ & \\
\hline URANO & 介 & \\
\hline NEPTUNO & $\Uparrow \quad \Omega$ & \\
\hline PLUTON & 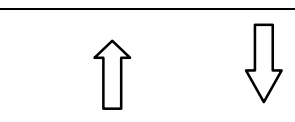 & \\
\hline $\begin{array}{l}\text { ¿Qué pasaría aquí? } \\
\text { SOL }\end{array}$ & 仓 & \\
\hline
\end{tabular}

